\title{
EDITORIAL
}

\section{Combination VEGFR/immune checkpoint inhibitor therapy: a promising new treatment for renal cell carcinoma}

\author{
British Journal of Cancer (2018) 119:911-912; https://doi.org/10.1038/s41416-018-0175-x
}

\section{SUMMARY}

Renal cell carcinoma is often managed with tyrosine kinase inhibitors and immune checkpoint inhibitors as monotherapy. Initial studies combining these two therapeutic strategies have demonstrated promising efficacy with manageable toxicity profiles. These combinations represent a promising new approach for metastatic renal cell carcinoma.

\section{MAIN}

Multitargeted receptor tyrosine kinase inhibitors (TKI) that inhibit the vascular endothelial growth factor receptor (VEGFR) are the mainstay of treatment for advanced/metastatic renal cell carcinoma (RCC). Although responses are frequent, most patients ultimately progress on their disease. Immune checkpoint inhibitors (ICI) targeting the PD-1/PD-L1 axis are active in RCC, but with only a fraction of patients achieving durable responses. Multiple ongoing clinical trials are currently exploring the safety and efficacy of combined VEGFR/ICI blockade in advanced/metastatic RCC.

In earlier clinical trials of dual VEGFR/ICI blockade, such as the combination of pembrolizumab/pazopanib, nivolumab/sunitinib, and nivolumab/pazopanib ${ }^{1,2}$ the efficacy was promising; however, the proportion of patients experiencing high grade toxicity discouraged further development. In the combinations of nivolumab/sunitinib and nivolumab/pazopanib, $73 \%$ and $60 \%$ of patients experienced grade 3 or worse treatment-related toxicities, respectively. Furthermore, adverse events leading to treatment discontinuation were observed in $23 \%$ of the nivolumab/sunitinib arm and $20 \%$ in the nivolumab/pazopanib arm. Similarly, the pembrolizumab/pazopanib combination was associated with high levels of hepatotoxicity and drug discontinuation. These combinations were deemed not suitable for testing within phase 3 trials. In contrast, subsequent combination of newer TKIs with $\mathrm{ICl}$ have shown improved toxicity profiles, with multiple combinations being investigated in phase 3 clinical trials including axitinib/ pembrolizumab, lenvatinib/pembrolizumab, cabozantinib/nivolumab, and axitinib/avelumab (Table 1). These TKls have a lower incidence of hepatoxicity, which may allow them to combine better with $\mathrm{ICl}$ blockade.

In a recent issue published in Lancet Oncology, Atkins et al. ${ }^{3}$ (2018) report the results of a phase $1 \mathrm{~b}$ trial of the combination of axitinib and pembrolizumab, in patients with advanced RCC. Fiftytwo previously untreated patients were included in a combined dose-finding (11 patients) and dose-expansion (41 patients) analysis. The cohorts consisted of 24 (46\%) international metastatic RCC database consortium (IMDC) favourable risk, 23 (44\%) intermediate risk, 3 (6\%) poor risk, and 2 (4\%) unknown risk patients. Treatment was well tolerated, with 34 (65\%) patients experiencing a grade 3 or worse treatment-related adverse event, and 28 (54\%) patients having a treatment-related severe adverse event. These toxicities were well manageable with dose modification, and consistent with what is seen with single agent TKI therapy. An objective response (partial or complete) was seen in 38 (73\%; 95\% Cl 59.0-84.4\%) patients. Median progression free survival was 20.9 months ( $95 \% \mathrm{Cl} 15.4-$ not evaluable), and the median overall survival was not reached with a median follow up period of 20.4 months.

Comparing the results of this phase $1 \mathrm{~b}$ trial to the results of others may not be feasible at this early stage, and cross trial efficacy comparison is challenging due to differences in patient population and patient context; therefore, these comparisons must always be considered with some caution. It must be noted that in this axitinib/ pembrolizumab trial, although the majority of patients were intermediate or poor risk, $46 \%$ of patients were IMDC favourable risk, which is higher than that typically seen for first line clinical trials. This trial demonstrated superior objective response rates compared to historic experiences with single agent tyrosine kinase inhibitors, which have typically ranged between 10 and $44 \%$ across several studies of various TKIs. Single agent axitinib was associated with a $32 \%$ objective response rate and a median progression free survival (PFS) of 10.3 months in a previous study in the first line setting ${ }^{4}$ however, the data for single agent PD-1 inhibition in the first line setting is limited. In patients previously treated with antiangiogenesis agents, PD-1 blockade with nivolumab was associated with a $25 \%$ objective response rate and a 4.6 month median PFS. Although a subgroup analysis of patients who did not progress within 6 months yielded a median PFS of 15.6 months $(95 \% \mathrm{Cl}, 11.8-19.6)$, suggesting that responses to $\mathrm{ICl}$ therapy could be extended. ${ }^{5}$

Promising efficacy data from other VEGF/ICI combinations have also been reported in RCC, including atezolizumab/ bevacizumab, ${ }^{6}$ avelumab/axitinib, ${ }^{7}$ and lenvatinib/pembrolizumab $^{8}$ (Table 1). IMmotion 151, a phase III clinical trial of bevacizumab in combination with atezolizumab was conducted in a risk population more similar to other RCC clinical trials. In that trial, only $20 \%$ of the intent-to-treat patients were favourable risk by MSKCC criteria, compared to $46 \%$ favourable risk by IMDC criteria in the axitinib/pembrolizumab trial reported by Atkins et al. These two risk criteria are well established and have high concordance. The primary endpoint was PFS in the PD-L1 positive patients, which showed an objective response rate of $43 \%(95 \% \mathrm{Cl} 35-50)$ and a median PFS of 11.2 months $(95 \% \mathrm{Cl} 8.9-15.0)$. In the phase $\mathrm{lb} / \mathrm{Il}$ assessing the combination of lenvatinib/pembrolizumab, both treatment naïve and pretreated patients were included, with $60 \%$ of patients being previously treated with one or more systemic therapies. In that study, the objective response rate was $63 \%$ (95\% Cl $44-80 \%)$ across the entire trial; after subgroup analysis of the treatment naïve cohort, the objective response rate rose 
Table 1. Select phase III clinical trials of $\mathrm{ICl}$ used in combination in RCC

\begin{tabular}{|c|c|c|c|}
\hline Clinical trial & Patients & Arms & Primary completion date \\
\hline CheckMate 214(NCT02231749) & 1096 & Nivolumab + ipilimumab $(n=550)$ vs sunitinib $(n=546)$ & June 26,2017 (reported) \\
\hline IMmotion151(NCT02420821) & 915 & Atezolizumab + bevacizumab $(n=454)$ vs sunitinib $(n=461)$ & December 2017(reported) \\
\hline JAVELIN Renal 101(NCT02684006) & 830(estimated) & Avelumab + axitinibvs sunitinib & December 2, 2018(estimated) \\
\hline E7080-G000-307(NCTNCT02811861) & 734(estimated) & $\begin{array}{l}\text { Lenvatinib }+ \text { pembrolizumab vsLenvatinib }+ \text { everolimus vs } \\
\text { sunitinib }\end{array}$ & January 15, 2020(estimated) \\
\hline KEYNOTE-426(NCT02853331) & 840(estimated) & Pembrolizumab + axitinibvs sunitinib & January 27, 2020(estimated) \\
\hline CheckMate 9ER(NCT03141177) & 1014(estimated) & Nivolumab + cabozantinibvs sunitinib & February 15, 2021(estimated) \\
\hline
\end{tabular}

to $83 \%(95 \% \mathrm{Cl} 52-98 \%)$. In this study $60 \%$ of patients experienced a Treatment Emergent Adverse Event, and the treatment was overall well tolerated with patients receiving $78 \%$ of the intended lenvatinib dose and $96 \%$ of the intended pembrolizumab dose. Progression free survival data remain to be reported for this trial.

Despite improved response rates, combination therapy is also associated with increased toxicity; therefore, biomarkers predictive for both response and resistance are critical for improving patient care and outcomes. However, biomarkers predicting response to combination ICI/VEGF remain poorly understood. In malignancies with a high mutational load, responses to $\mathrm{ICI}$ have been associated with tumour mutation burden and PD-1 status; ${ }^{9-11}$ it is currently unclear whether these biomarkers can be translated to low mutation-load malignancies, such as RCC. ${ }^{12}$ Furthermore, the correlation between total mutation burden and the number of mutations found by targeted exome sequencing have been established in predominately high mutation burden or carcinogen-driven malignancies, and it has yet to be established whether these estimates of tumour mutational burden are valid in RCC, which has a lower mutation rate and a higher proportion of insertion deletion mutations compared with other malignancies. It is also unclear whether biomarkers for single agent PD-1 inhibition or combination $\mathrm{ICl} / \mathrm{ICl}$ therapy remain relevant to $\mathrm{ICI} / V E G F$ therapy, and how combination therapy changes the thresholds for response to therapy.

PD-L1 status has also been widely investigated in RCC and other malignancies; however, its role as a biomarker in RCC remains controversial. Responses to $\mathrm{ICl}$ combinations such as ipilimumab/nivolumab have been correlated with PD-L1 status; $^{13}$ yet responses to axitinib/pembrolizumab were not. It remains to be seen whether concurrent TKI targeting decouples tumour responses from dependence on PD-L1 expression, 3,8 because high response levels are often seen in both PD-L1 positive and PD-L1 negative patients in VEGFR/ICI trials. More extensive studies are necessary to understand the biology and pathophysiology associated with combination ICI/VEGF targeted therapy.

Progression free survival data remain pending for other combinations of VEGFR/ICI therapy, and several phase III clinical trials are ongoing. The combination of axitinib/pembrolizumab presented by Atkins et al. shows promising efficacy with manageable tolerability and safety in this phase lb clinical trial, and is currently being further tested in a randomised phase III clinical trial (KEYNOTE-426) of axitinib/pembrolizumab vs sunitinib. Taken as a whole, the recent combinations of VEGFR/ICI therapy have shown encouraging results. With multiple combinations currently under investigation, it remains to be seen which of these combinations may be superior and how the VEGFR/ICI therapy approach compares to combination $\mathrm{ICl}$ therapy, such as ipilimu$\mathrm{mab} /$ nivolumab. Until head-to-head trials of these combinations can be performed to compare their efficacy, biomarker development will be critical for patient stratification.

\section{ADDITIONAL INFORMATION}

Competing interests: C.-H.L.: Consulting (Exelixis, Eisai), and Research Funds to the Institute (BMS, Exelixis, Pfizer, Calithera, Eisai). R.J.M.: Consulting (Novartis, Pfizer, Eisai, Exelixis), and research funds to the institute (Novartis, Pfizer, Eisai, Exelixis, BMS, Genetech/Roche).

Note: This work is published under the standard license to publish agreement. After 12 months the work will become freely available and the license terms will switch to a Creative Commons Attribution-NonCommercial-Share Alike 4.0 Unported License).

Chung-Han Lee and Robert Motzer

Department of Medicine, Genitourinary Oncology Service, Memorial Sloan Kettering Cancer Center, New York, NY, USA Correspondence: C-H. Lee (Leec4@mskcc.org)

\section{REFERENCES}

1. Chowdhury, S. et al. A phase I/II study to assess the safety and efficacy of pazopanib (PAZ) and pembrolizumab. (PEM) Patients (pts) Adv. Ren. Cell Carcinoma 35, 4506 (2017).

2. Amin, A. et al. Nivolumab (anti-PD-1; BMS-936558, ONO-4538) in combination with sunitinib or pazopanib in patients (pts) with metastatic renal cell carcinoma (mRCC). Nivolumab (anti-PD-1; BMS-936558, ONO-4538) in combination with sunitinib or pazopanib in patients (pts) with metastatic renal cell carcinoma (mRCC), Journal of Clinical Oncology. 32, (suppl.), abstr. 5010 (2014).

3. Atkins, M. B. et al. Axitinib in combination with pembrolizumab in patients with advanced renal cell cancer: a non-randomised, open-label, dose-finding, and dose-expansion phase 1b trial. Lancet Oncol. 19, 405-415 (2018).

4. Hutson, T. E. et al. Comparing the results of this Phase $1 \mathrm{~b}$ trial to the results of others may not be feasible at this early stage. Lancet Oncol. 14, 1287-1294 (2013).

5. Motzer, R. J. et al. Nivolumab versus everolimus in advanced renal-cell carcinoma. N. Engl. J. Med. 373, 1803-1813 (2015).

6. Motzer, R. J. et al. IMmotion151: a randomized phase III study of atezolizumab plus bevacizumab vs sunitinib in untreated metastatic renal cell carcinoma (mRCC), Journal of Clinical Oncology. 36, abstr. 578 (2018).

7. Choueiri, T. K. et al. First-line avelumab + axitinib therapy in patients (pts) with advanced renal cell carcinoma (aRCC): results from a phase lb trial, Journal of Clinical Oncology. 35, 4504 (2017).

8. Lee, $C$. et al. A phase $1 \mathrm{~b} / 2$ trial of lenvatinib and pembrolizumab in patients with renal cell carcinoma. In Oral presentation at the 42nd European Society of Medical Oncology (ESMO) Annual Meeting (2017). https://doi.org/10.1093/annonc/ $\mathrm{md} \times 371.002$

9. Rizvi, N. A. et al. Cancer immunology. Mutational landscape determines sensitivity to PD-1 blockade in non-small cell lung cancer. Science 348, 124-128 (2015).

10. Snyder, A. et al. Genetic basis for clinical response to CTLA-4 blockade in melanoma. N. Engl. J. Med. 371, 2189-2199 (2014).

11. Van Allen, E. M. et al. Genomic correlates of response to CTLA-4 blockade in metastatic melanoma. Science 350, 207-211 (2015).

12. Turajlic, S. et al. Insertion-and-deletion-derived tumour-specific neoantigens and the immunogenic phenotype: a pan-cancer analysis. Lancet Oncol. 18, 1009-1021 (2017).

13. Escudier, B. et al. CheckMate 214: efficacy and safety of nivolumab plus ipilimumab vs sunitinib for treatment-naive advanced or metastatic renal cell carcinoma, including IMDC risk and PD-L1 expression subgroups. In Oral presentation at the 42nd European Society of Medical Oncology (ESMO) Annual Meeting (2017). https:// doi.org/10.1093/annonc/mdx440.029 